\title{
Looking at aneurysms by the numbers: morphometrics and the potentials of aneurysm analysis
}

\author{
Charles J. Prestigiacomo, MD \\ Department of Neurological Surgery, University of Cincinnati College of Medicine, Cincinnati, Ohio
}

$\mathrm{P}$ REDICTING aneurysm rupture, to many, is the "holy grail" for cerebrovascular specialists. Aneurysms and their sequelae affect our patients during some of their most productive years of life and with often times debilitating or deadly consequences. In the last two decades, the power of the desktop computer has brought about a rapid evolution in the application of morphometrics and biophysics to the study of vascular systems, such that the analysis of aneurysm formation, growth, and rupture has expanded beyond observational characteristics and incorporated many aspects of flow dynamics.

Understanding the biophysical nature of aneurysms is inferred. No current study or in vivo analysis has yet been developed to allow clinicians to accurately predict the rupture status of aneurysms, let alone accurately predict which aneurysms are at risk for future rupture. Consequently, several surrogate markers have been studied and have served as the initial parameters in helping to understand aneurysmal behavior.

Biomorphometric studies demonstrate relationships between various observable, physical characteristics of aneurysms and their parent vessels and rupture status. These techniques provide some of the most straightforward analyses of aneurysms. Indeed, early studies assessing size, shape, and location established the basic tenets associated with aneurysm rupture: tenets that have been validated through numerous additional studies. ${ }^{1-3,8,10,12}$

As discussed, though these primary (measurements obtained directly as measured from imaging studies) and secondary, derived analyses (results as calculated from basic measurements) have correlated with rupture, they have only served as surrogates and not true predictive models because of the significant ethical concerns that exist in prospectively following patients who may succumb to the sequelae of rupture. ${ }^{4-6,9,11}$

Though much has been written about the formation of aneurysms and a fair amount written about the rup- ture of these lesions, an understanding of growth, one of the more critical characteristics of cerebral aneurysms, is somewhat elusive. Leemans et al. ${ }^{7}$ provide insight into this very difficult aspect of aneurysm behavior by specifically studying morphometric relationships in aneurysms with demonstrable growth. Their retrospective analysis of 38 aneurysms in 31 patients assessed 12 morphological characteristics related to changes in aneurysm size and shape during a period that ranged from 23 to 567 weeks. Though they detected statistical significance in all sizerelated parameters, as might be expected, they also noted that some shape indices, such as the bottleneck factor, significantly increased, while the ellipticity index significantly decreased. Taken together, these data suggested that as the aneurysm grows, it trends toward forming a larger and larger sphere. An intriguing observation and a limitation of this discussion revolve around the fact that 12 of the growing aneurysms were treated after growth was documented because of significant changes in shape. One ruptured during the follow-up period after documented growth.

These data, as with all good science, leave us with more questions in the midst of some insights and answers. Though it is clear from the data that growth occurs in multiple dimensions and that larger aneurysms are associated with rupture, this very growth is not equal along all axes. However, does the rate of growth affect the way the growth occurs and is the rate of growth more dangerous than any other parameter measured? Does an aneurysm form and grow toward a shape of a sphere, and after a certain size does it begin to develop irregularities such that shape becomes the dominant characteristic of rupture as opposed to size? Does this correlate with certain demographic characteristics of the patient? How many aneurysms remain unchanged and why? These are but a few salient questions that emerge from this study.

This study brings together the many aspects involved 
in today's study of aneurysms and demonstrates the multidisciplinary nature from which meaningful data are obtained. It also brings to this readership a glimpse of what can be gleaned from the data that exists in every large series of patients. By creating collaborative efforts between experts in the various fields of mathematics, physics, and engineering and clinicians in neuroimaging, neurology, and neurosurgery, new insights are developed that may bring us closer to solving the riddle of the aneurysm. No datum is "too simplistic" and no data series is "too complex" for analysis.

Are we thus any closer to predicting aneurysm rupture now than 20 years ago? Are the techniques that are being developed to study growth and rupture helpful in understanding recurrence after treatment? Clearly, understanding the biophysics of aneurysm formation is a stepping-stone to understanding the efficacy of aneurysm treatment. In conjunction with studies in the genetics, epidemiology, and pathobiology of aneurysm formation, studying the morphometry and biophysics of aneurysm formation provides a more complete picture of how these factors interact. What should follow is the development of an albeit complex algorithm that would allow multiple input parameters obtained from images, serological studies, and genetic testing that would provide a relative rupture risk for a patient-specific aneurysm, what some call "big data" and the machine learning algorithms involved in integrating such disparate data sets into a cohesive, interpretable and meaningful output. In so doing, the physician would thus be given an important tool to help guide the patient toward the best option for that patient with regard to treatment or observation. Indeed, such software and its algorithms would likewise enable clinicians to determine best treatment patterns or perhaps simulate the best therapy and assess long-term efficacy. After all, exposing only those patients at a true risk of rupture to treatment, and its devastating sequelae, is what all clinicians strive for.

https://thejns.org/doi/abs/10.3171/2019.4.FOCUS19332

\section{References}

1. Asari S, Ohmoto T: Natural history and risk factors of unruptured cerebral aneurysms. Clin Neurol Neurosurg 95:205-214, 1993

2. Backes D, Rinkel GJE, Laban KG, Algra A, Vergouwen MDI: Patient- and aneurysm-specific risk factors for intracranial aneurysm growth: a systematic review and metaanalysis. Stroke 47:951-957, 2016
3. Dickey P, Kailasnath P: The diameter-cube hypothesis: a new biophysical model of aneurysm rupture. Surg Neurol 58:166-173, 2002

4. Ghosh S, Dey S, Tjoumakaris S, Gonzalez F, Rosenwasser R, Pascal J, et al: Association of morphologic and demographic features of intracranial aneurysms with their rupture: a retrospective analysis. Acta Neurochir Suppl 115:275-278, 2013

5. Hoh BL, Sistrom CL, Firment CS, Fautheree GL, Velat GJ, Whiting I, et al: Bottleneck factor and height-width ratio: association with ruptured aneurysms in patients with multiple cerebral aneurysms. Neurosurgery 61:716-722, 2007

6. Inagawa T, Hirano A: Autopsy study of unruptured incidental intracranial aneurysms. Surg Neurol 34:361-365, 1990

7. Leemans EL, Cornelissen BMW, Said M, van den Berg R, Slump CH, Marquering HA, et al: Intracranial aneurysm growth: consistency of morphological changes. Neurosurg Focus 47(1):E5, 2019

8. Ma B, Harbaugh RE, Raghavan ML: Three-dimensional geometrical characterization of cerebral aneurysms. Ann Biomed Engineering 32:264-273, 2004

9. Nikolic I, Tasic G, Bogosavljevic V, Nestorovic B, Jovanovic V, Kojic Z, et al: Predictable morphometric parameters for rupture of intracranial aneurysms - a series of 142 operated aneurysms. Turk Neurosurg 22:420-426, 2012

10. Omodaka S, Sugiyama S, Inoue T, Funamoto K, Fujimura M, Shimizu H, et al: Local hemodynamics at the rupture point of cerebral aneurysms determined by computational fluid dynamics analysis. Cerebrovasc Dis 34:121-129, 2012

11. Prestigiacomo CJ, He W, Catrambone J, Chung S, Kasper L, Pasupuleti L, et al: Predicting aneurysm rupture probabilities through the application of a computed tomography angiography-derived binary logistic regression model. J Neurosurg 110:1-6, 2009

12. Raghavan ML, Ma B, Harbaugh RE: Quantified aneurysm shape and rupture risk. J Neurosurg 102:355-362, 2005

\section{Disclosures}

Dr. Prestigiacomo serves on the boards of the following: Aesculap, Thermopeutix, Cerenovus, and the International Brain Research Foundation.

\section{Correspondence}

Charles J. Prestigiacomo: presticj@uc.edu.

\section{INCLUDE WHEN CITING}

DOI: 10.3171/2019.4.FOCUS19332. 